\title{
Paenibacillus xylanisolvens sp. nov., a xylan- degrading bacterium from soil
}

\author{
Saowapar Khianngam, ${ }^{1}$ Somboon Tanasupawat, ${ }^{1}$ \\ Ancharida Akaracharanya, ${ }^{2}$ Kwang Kyu Kim, ${ }^{3}$ Keun Chul Lee ${ }^{3}$ \\ and Jung-Sook Lee ${ }^{3}$
}

Correspondence

Somboon Tanasupawat

Somboon.T@chula.ac.th

Jung-Sook Lee

jslee@kribb.re.kr

\author{
1Department of Biochemistry and Microbiology, Faculty of Pharmaceutical Sciences, Chulalongkorn \\ University, Bangkok 10330, Thailand \\ ${ }^{2}$ Department of Microbiology, Faculty of Science, Chulalongkorn University, Bangkok 10330, \\ Thailand \\ ${ }^{3}$ Korean Collection for Type Cultures, Biological Resource Center, Korea Research Institute of \\ Bioscience and Biotechnology, Yuseong, Daejeon 305-806, Republic of Korea
}

\begin{abstract}
A xylan-degrading bacterium, strain $\mathrm{X} 11^{\top}{ }^{\top}$, was isolated from soil collected in Nan province, Thailand. The strain was characterized based on its phenotypic and genotypic characteristics. Strain $\mathrm{X} 11-1^{\top}$ was a Gram-stain-positive, facultatively anaerobic, spore-forming, rod-shaped bacterium. It contained meso-diaminopimelic acid in the cell-wall peptidoglycan. The major menaquinone was MK-7, anteiso- $C_{15: 0}(56.6 \%)$ and $C_{16: 0}(14.0 \%)$ were the predominant cellular fatty acids and diphosphatidylglycerol, phosphatidylmonomethylethanolamine, phosphatidylethanolamine and phosphatidylglycerol were the major phospholipids. The DNA $\mathrm{G}+\mathrm{C}$ content was $51.6 \mathrm{~mol} \%$. Phylogenetic analysis using $16 \mathrm{~S}$ rRNA gene sequences showed that strain $\mathrm{X} 11-1^{\top}$ was affiliated to the genus Paenibacillus and was closely related to Paenibacillus naphthalenovorans KACC $11505^{\top}$ and Paenibacillus validus CCM $3894^{\top}$, with $96.5 \%$ sequence similarity. Therefore, the strain represents a novel species of the genus Paenibacillus, for which the name Paenibacillus xylanisolvens sp. nov. is proposed. The type strain is $\mathrm{X} 11-1^{\top}\left(=\right.$ KCTC $13042^{\top}=$ PCU $311^{\top}=$ TISTR $\left.1829^{\top}\right)$.
\end{abstract}

The genus Paenibacillus, proposed by Ash et al. (1993, 1994), has undergone considerable changes, with a consequent increase in the number of species. At the time of writing, there are 112 recognized species (Euzéby, 2010). Paenibacillus strains have been isolated from diverse environmental samples such as soil, cow faeces, dead honeybees and honeybee larvae, plant roots, food, a warm spring, raw and heat-treated milk and blood cultures (Shida et al., 1997; Berge et al., 2002; Roux \& Raoult, 2004; Scheldeman et al., 2004; Velázquez et al., 2004; Chou et al., 2007). Some of these bacteria excrete a diverse assortment of extracellular enzymes that hydrolyse polysaccharides (Zamost et al., 1991; Morales et al., 1995; Hespell, 1996; Nielsen \& Sørensen, 1997; Aÿ et al., 1998; Lee et al., 2000; Dasman et al., 2002; Kim et al., 2004). Among them, strains of Paenibacillus barcinonensis, P. cellulosilyticus, P. favisporus, $P$. montaniterrae, $P$. nanensis, $P$. panacisoli, P. phyllosphaerae, P. septentrionalis, P. siamensis, P. soli,

The GenBank/EMBL/DDBJ accession number for the 16S rRNA gene sequence of strain $\times 11-1^{\top}$ is AB495094.

Three supplementary figures are available with the online version of this paper.
P. thailandensis, $P$. tundrae, $P$. woosongensis, $P$. xylanexedens and P. xylanilyticus have been reported to have xylandegrading activity (Velázquez et al., 2004; Rivas et al., 2005a, b, 2006; Sánchez et al., 2005; Ten et al., 2006; Park et al., 2007; Lee \& Yoon, 2008; Khianngam et al., 2009a, b; Nelson et al., 2009). In this paper, we describe a novel xylan-degrading bacterium belonging to the genus Paenibacillus by means of a polyphasic taxonomic study.

Strain X11-1 ${ }^{\mathrm{T}}$ was isolated from a soil sample collected in Viengsa district, Nan province, in the northern part of Thailand, by the spread-plate method on XC agar medium $\left(1^{-1}: 10\right.$ g oat spelt xylan, 5 g peptone, $1 \mathrm{~g}$ yeast extract, $4 \mathrm{~g}$ $\mathrm{K}_{2} \mathrm{HPO}_{4}, 1 \mathrm{~g} \mathrm{MgSO}_{4} .7 \mathrm{H}_{2} \mathrm{O}, 0.2 \mathrm{~g} \mathrm{KCl}, 0.02 \mathrm{~g} \mathrm{FeSO}_{4}$. $7 \mathrm{H}_{2} \mathrm{O}, 15 \mathrm{~g}$ agar; $\mathrm{pH}$ 7.0). In this screening step, the agar plates were incubated at $40{ }^{\circ} \mathrm{C}$ for 2 days. Xylan-degrading capacity of the cultures was detected by using a Congo red overlay method, as reported previously (Teather \& Wood, 1982; Ruijssenaars \& Hartsmans, 2001). Isolates that showed xylanase production were transferred to $\mathrm{C}$ agar medium. This medium had the same composition as XC medium apart from the omission of the oat spelt xylan. Cells grown on $\mathrm{C}$ agar medium were examined for their morphological and cultural characteristics, including cell 
shape, colony appearance, endospore formation and pigmentation, after incubation at $37{ }^{\circ} \mathrm{C}$ for 2 days. Motility was observed under a phase-contrast microscope (Nikon ECLIPSE $80 i$ ) by the hanging-drop method. Physiological and biochemical characterization was performed using the API 20NE and API 50CH (combined with API 50CHB/E medium) strips (bioMérieux), in accordance with the manufacturer's directions. Tests for Gram staining, catalase and oxidase, hydrolysis of casein, DNA, starch, Tween 80 and L-tyrosine, the methyl red/Voges-Proskauer reactions, indole production, citrate utilization and $\mathrm{H}_{2} \mathrm{~S}$ production were determined as described by Barrow \& Feltham (1993). Growth under anaerobic conditions on agar plates was investigated using a Gaspak (BBL) anaerobic jar. Growth at $\mathrm{pH} 5,6,7,8$ and 9 , in 3 and $5 \%(\mathrm{w} / \mathrm{v}) \mathrm{NaCl}$ and at 10,15 , $20,25,30,40,45,50,55$ and $60{ }^{\circ} \mathrm{C}$ was tested by using $\mathrm{C}$ agar medium. All tests were carried out by incubating the cultures at $37{ }^{\circ} \mathrm{C}$, except for the investigation of the effect of temperature on growth.

The type of diaminopimelic acid in the cell wall and menaquinones were determined as described by Komagata \& Suzuki (1987). Total cellular fatty acid analysis was performed with cells grown on tryptic soy agar (TSA; Difco) for $48 \mathrm{~h}$ at $30{ }^{\circ} \mathrm{C}$, by using the standard Microbial Identification System (MIDI Inc.) for automated GC analysis (Sasser, 1990). Polar lipids were extracted, examined by two-dimensional TLC and identified by using published procedures (Minnikin et al., 1977).

DNA was prepared by the method of Saito \& Miura (1963). DNA base composition was determined by reversed-phase HPLC (Tamaoka \& Komagata, 1984). The 16 S rRNA gene of the novel strain was amplified and the PCR product was purified and sequenced as described previously (Tanasupawat et al., 2004). The sequence of strain X11-1 ${ }^{\mathrm{T}}(1472 \mathrm{nt})$ was aligned with selected sequences obtained from GenBank by using CLUSTAL_X version 1.83 (Thompson et al., 1997). The alignment was edited manually to remove gaps and ambiguous nucleotides prior to the construction of phylogenetic trees. Phylogenetic trees were constructed by using the neighbour-joining (Saitou \& Nei, 1987) and maximumparsimony (Fitch, 1971) methods in the MEGA4 software (Tamura et al., 2007). Confidence values of branches of the trees were determined using bootstrap analysis (Felsenstein, 1985) based on 1000 resamplings.

Strain $\mathrm{X} 11-1^{\mathrm{T}}$ was a Gram-stain-positive, facultatively anaerobic, motile, rod-shaped bacterium. Central or subterminal ellipsoidal endospores were observed in swollen sporangia (Supplementary Fig. S1, available in IJSEM Online). Colonies were $1-5 \mathrm{~mm}$ in diameter, circular, flat, white and opaque after incubation on $\mathrm{C}$ medium for 2 days. Phenotypic characteristics are listed in the species description and in Table 1. Strain $\mathrm{X}_{11-1^{\mathrm{T}}}$ contained anteiso- $\mathrm{C}_{15: 0}$ $(56.6 \%)$ and $\mathrm{C}_{16: 0}(14.0 \%)$ as the predominant cellular fatty acids. Strain X11-1 ${ }^{\mathrm{T}}$, Paenibacillus naphthalenovorans KACC $11505^{\mathrm{T}}$ and Paenibacillus validus CCM $3894^{\mathrm{T}}$ showed similar cellular fatty acid profiles, but significant quantitative
Table 1. Differential characteristics of strain $X 11-1^{\top}$ and closely related Paenibacillus type strains

Strains: $1, \mathrm{X} 11-1^{\mathrm{T}}$; 2, P. naphthalenovorans KACC $11505^{\mathrm{T}}$; 3, P. validus CCM $3894^{\mathrm{T}}$. Data were obtained in this study unless otherwise indicated. +, Positive; w, weakly positive; - , negative.

\begin{tabular}{|c|c|c|c|}
\hline Characteristic & 1 & 2 & 3 \\
\hline Growth in $3 \% \mathrm{NaCl}$ & $\mathrm{w}$ & $\mathrm{W}$ & - \\
\hline \multicolumn{4}{|l|}{ Growth at: } \\
\hline $15{ }^{\circ} \mathrm{C}$ & + & - & $\mathrm{w}$ \\
\hline $50{ }^{\circ} \mathrm{C}$ & + & + & - \\
\hline $55^{\circ} \mathrm{C}$ & - & + & - \\
\hline Voges-Proskauer reaction & - & + & - \\
\hline Nitrate reduction & + & - & + \\
\hline \multicolumn{4}{|l|}{ Hydrolysis of: } \\
\hline Aesculin & $\mathrm{w}$ & - & + \\
\hline Casein & + & - & - \\
\hline Starch & - & - & + \\
\hline Assimilation of potassium gluconate & - & + & + \\
\hline \multicolumn{4}{|l|}{ Acid production from: } \\
\hline Amygdalin & - & - & + \\
\hline D-Arabinose & + & + & - \\
\hline D-Arabitol & + & - & - \\
\hline Cellobiose & - & - & + \\
\hline Glycerol & + & - & + \\
\hline Glycogen & - & - & + \\
\hline Inositol & - & - & + \\
\hline D-Lyxose & + & + & - \\
\hline Melibiose & - & + & + \\
\hline Raffinose & - & + & + \\
\hline Starch & - & + & + \\
\hline Xylitol & - & + & - \\
\hline DNA G $+\mathrm{C}$ content $(\mathrm{mol} \%)$ & 51.6 & $46.6^{a_{\star}}$ & $50.9^{b}$ \\
\hline
\end{tabular}

${ }^{\star}$ Data from: $a$, Daane et al. (2002); b, Heyndrickx et al. (1995).

differences were also found, as shown in Table 2. In the $16 \mathrm{~S}$ rRNA gene sequence-based phylogenetic tree reconstructed according to the neighbour-joining method, strain X11- ${ }^{\mathrm{T}}$ was placed in a monophyletic cluster of closely related Paenibacillus species (Fig. 1). Trees constructed by the neighbour-joining (Supplementary Fig. S2) and maximumparsimony (not shown) methods including strain X11-1 ${ }^{\mathrm{T}}$ and representatives of all Paenibacillus species were compared and strain X11-1 ${ }^{\mathrm{T}}$ was closely related to $P$. naphthalenovorans $\mathrm{PR}-\mathrm{N} 1^{\mathrm{T}}, P$. validus $\mathrm{JCM} 9077^{\mathrm{T}}$, P. ehimensis KCTC $3748^{\mathrm{T}}$, P. elgii $\mathrm{SD} 17^{\mathrm{T}}$, P. koreensis $\mathrm{YC} 00^{\mathrm{T}}$, P. soli $\mathrm{DCY}^{\mathrm{T}}{ }^{\mathrm{T}}$ and $P$. filicis $S 4^{\mathrm{T}}$ based on $16 \mathrm{~S}$ rRNA gene sequence similarity (less than $97 \%$ ). The DNA G $+C$ content was $51.6 \mathrm{~mol} \%$, which was in the range observed for members of the genus Paenibacillus (Shida et al., 1997). It contained meso-diaminopimelic acid in the cell-wall peptidoglycan, the major menaquinone was MK-7 and the major phospholipids were diphosphatidylglycerol, phosphatidylmonomethylethanolamine, phosphatidylethanolamine and phosphatidylglycerol (Supplementary Fig. S3); glycolipids were not detected in this study. Based on its phenotypic and chemotaxonomic 
Table 2. Cellular fatty acid compositions of strain $X 11-1^{\top}$ and closely related Paenibacillus type strains

Strains: $1, \mathrm{X} 11-1^{\mathrm{T}}$; 2, P. naphthalenovorans KACC $11505^{\mathrm{T}} ; 3, P$. validus CCM $3894^{\mathrm{T}}$. Data were obtained in this study. Values are percentages of total fatty acids. tr, Trace $(<1 \%)$; ND, not detected.

\begin{tabular}{|c|c|c|c|}
\hline Fatty acid & 1 & 2 & 3 \\
\hline \multicolumn{4}{|c|}{ Straight-chain saturated } \\
\hline $\mathrm{C}_{12: 0}$ & ND & ND & $\operatorname{tr}$ \\
\hline $\mathrm{C}_{14: 0}$ & 2.4 & 2.2 & 2.0 \\
\hline $\mathrm{C}_{15: 0}$ & 3.1 & $\operatorname{tr}$ & $\operatorname{tr}$ \\
\hline $\mathrm{C}_{16: 0}$ & 14.0 & 17.2 & 15.2 \\
\hline $\mathrm{C}_{17: 0}$ & $\operatorname{tr}$ & $\mathrm{ND}$ & $\operatorname{tr}$ \\
\hline \multicolumn{4}{|c|}{ Branched saturated } \\
\hline iso- $\mathrm{C}_{14: 0}$ & 2.4 & 1.2 & 1.3 \\
\hline iso- $\mathrm{C}_{15: 0}$ & 5.1 & 6.5 & 9.9 \\
\hline iso- $\mathrm{C}_{16: 0}$ & 7.1 & 8.8 & 6.9 \\
\hline iso- $\mathrm{C}_{17: 0}$ & 1.7 & 4.5 & 7.7 \\
\hline anteiso- $\mathrm{C}_{15: 0}$ & 56.6 & 47.2 & 44.6 \\
\hline anteiso- $\mathrm{C}_{17: 0}$ & 5.2 & 9.3 & 9.7 \\
\hline \multicolumn{4}{|l|}{ Monounsaturated } \\
\hline $\mathrm{C}_{16: 1} \omega 7 c$ alcohol & ND & $\mathrm{ND}$ & $\operatorname{tr}$ \\
\hline $\mathrm{C}_{16: 1} \omega 11 c$ & 1.1 & 2.5 & $\operatorname{tr}$ \\
\hline iso- $\mathrm{C}_{17: 1} \omega 10 c$ & ND & ND & $\operatorname{tr}$ \\
\hline
\end{tabular}

characteristics and phylogenetic analysis using 16S rRNA gene sequences, we conclude that strain X11-1 ${ }^{\mathrm{T}}$ represents a novel species of the genus Paenibacillus, for which the name Paenibacillus xylanisolvens sp. nov. is proposed.

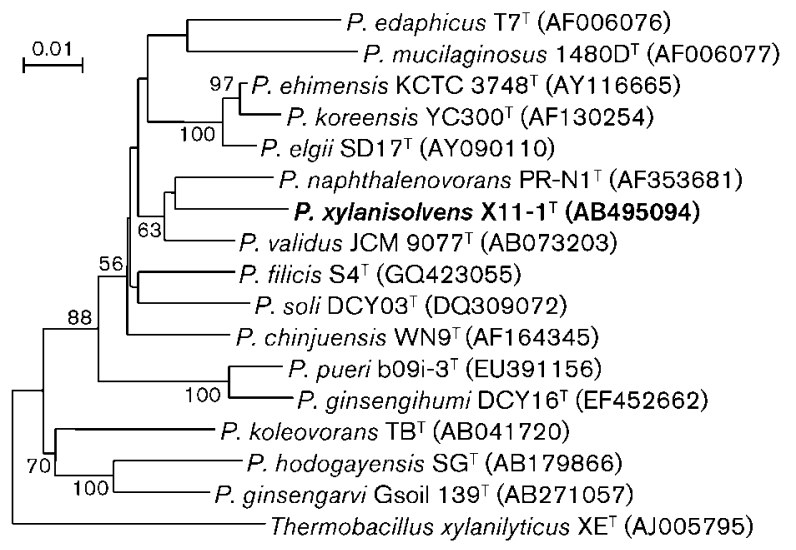

Fig. 1. Neighbour-joining tree based on 16S rRNA gene sequences showing the phylogenetic relationships between strain $\mathrm{X} 11-1^{\top}$ and the type strains of closely related Paenibacillus species. Thermobacillus xylanilyticus $\mathrm{XE}^{\top}$ was used as the outgroup. Based on 1000 resamplings, bootstrap percentages $\geqslant 56 \%$ are shown. Bar, 0.01 substitutions per nucleotide position. An extended neighbour-joining tree is available as Supplementary Fig. S2.

\section{Description of Paenibacillus xylanisolvens sp. nov.}

Paenibacillus xylanisolvens (xy.la.ni.sol'vens. N.L. n. xylanum xylan; L. part. adj. solvens dissolving; N.L. part. adj. xylanisolvens xylan-dissolving).

Cells are Gram-stain-positive, facultatively anaerobic, motile rods $(0.4-0.8 \times 1.5-3.0 \mu \mathrm{m})$. Central or subterminal ellipsoidal endospores are observed in swollen sporangia. Colonies are $1-5 \mathrm{~mm}$ in diameter, circular, flat, white and opaque after 2 days of incubation on $\mathrm{C}$ agar medium. Growth is observed at $\mathrm{pH} \mathrm{6-9} \mathrm{(optimally} \mathrm{at} \mathrm{pH} 7-8$ ), at $15-50{ }^{\circ} \mathrm{C}$ (optimally at $30-37{ }^{\circ} \mathrm{C}$ ), in $3 \% \mathrm{NaCl}$ (weakly) and under anaerobic conditions, but not at $\mathrm{pH} 5$, at 10, 55 or $60{ }^{\circ} \mathrm{C}$ or in $5 \% \mathrm{NaCl}$. Positive for catalase, oxidase, nitrate reduction, hydrolysis of aesculin (weak), casein, Tween 80, urea and xylan and assimilation of D-glucose, maltose, D-mannitol and D-mannose, but negative for methyl red and Voges-Proskauer reactions, citrate utilization, $\mathrm{H}_{2} \mathrm{~S}$ production, indole production, fermentation of glucose, hydrolysis of L-arginine, DNA, gelatin, $p$-nitrophenyl $\beta$-D-galactopyranoside, starch and L-tyrosine and assimilation of $\mathrm{N}$-acetylglucosamine, L-arabinose, adipic acid, capric acid, phenylacetic acid and potassium gluconate. Able to produce acid from aesculin, D-arabinose, D-arabitol, D-fructose, $\alpha$-glucopyranoside, D-galactose, Dglucose, glycerol, D-lyxose, maltose, D-mannitol, D-mannose, D-ribose, sucrose, trehalose, turanose and D-xylose, but not from $\mathrm{N}$-acetylglucosamine, D-adonitol, amygdalin, L-arabinose, L-arabitol, arbutin, cellobiose, dulcitol, erythritol, D- or L-fucose, gentiobiose, gluconate, glycogen, inositol, inulin, 2ketogluconate, 5-ketogluconate, lactose, $\alpha$-mannopyranoside, melezitose, melibiose, raffinose, L-rhamnose, salicin, Dsorbitol, L-sorbose, starch, D-tagatose, $\beta$-xylopyranoside, xylitol or L-xylose. MK-7 is the predominant menaquinone. The predominant fatty acids are anteiso- $\mathrm{C}_{15: 0}$ and $\mathrm{C}_{16: 0}$. The cell-wall diamino acid is meso-diaminopimelic acid. Major phospholipids are diphosphatidylglycerol, phosphatidylmonomethylethanolamine, phosphatidylethanolamine and phosphatidylglycerol. The DNA G $+\mathrm{C}$ content of the type strain is $51.6 \mathrm{~mol} \%$.

The type strain is X11-1 $1^{\mathrm{T}}\left(=\right.$ KCTC $13042^{\mathrm{T}}=\mathrm{PCU} 311^{\mathrm{T}}$ $=$ TISTR $1829^{\mathrm{T}}$ ), isolated from soil.

\section{Acknowledgements}

A Government Research Grant, Chulalongkorn University (2005), a scholarship from the Royal Golden Jubilee Ph.D. Program (2007) to S. K. and a grant from the KRIBB Research Initiative Program are gratefully acknowledged.

\section{References}

Ash, C., Priest, F. G. \& Collins, M. D. (1993). Molecular identification of rRNA group 3 bacilli (Ash, Farrow, Wallbanks and Collins) using a PCR probe test. Antonie van Leeuwenhoek 64, 253-260.

Ash, C., Priest, F. G. \& Collins, M. D. (1994). Paenibacillus gen. nov. In Validation of the Publication of New Names and New Combinations 
Previously Effectively Published Outside the IJSB, List no. 51. Int J Syst Bacteriol 44, 852-853.

Aÿ, J., Goetz, F., Borriss, R. \& Heinemann, U. (1998). Structure and function of the Bacillus hybrid enzyme GluXyn-1: native-like jellyroll fold preserved after insertion of autonomous globular domain. Proc Natl Acad Sci U S A 95, 6613-6618.

Barrow, G. I. \& Feltham, R. K. A. (1993). Cowan and Steel's Manual for the Identification of Medical Bacteria, 3rd edn. Cambridge: Cambridge University Press.

Berge, O., Guinebretière, M. H., Achouak, W., Normand, P. \& Heulin, T. (2002). Paenibacillus graminis sp. nov. and Paenibacillus odorifer sp. nov., isolated from plant roots, soil and food. Int J Syst Evol Microbiol 52, 607-616.

Chou, J. H., Chou, Y. J., Lin, K. Y., Sheu, S. Y., Sheu, D. S., Arun, A. B., Young, C. C. \& Chen, W. M. (2007). Paenibacillus fonticola sp. nov., isolated from a warm spring. Int J Syst Evol Microbiol 57, 1346-1350.

Daane, L. L., Harjono, I., Barns, S. M., Launen, L. A., Palleroni, N. J. \& Häggblom, M. M. (2002). PAH-degradation by Paenibacillus spp. and description of Paenibacillus naphthalenovorans sp. nov., a naphthalene-degrading bacterium from the rhizosphere of salt marsh plants. Int J Syst Evol Microbiol 52, 131-139.

Dasman, Kajiyama, S., Kawasaki, H., Yagi, M., Seki, T., Fukusaki, E. \& Kobayashi, A. (2002). Paenibacillus glycanilyticus sp. nov., a novel species that degrades heteropolysaccharide produced by the cyanobacterium Nostoc commune. Int J Syst Evol Microbiol 52, 1669-1674.

Euzéby, J. P. (2010). Genus Paenibacillus. In List of Prokaryotic Names with Standing in Nomenclature. Last full update 16 February 2010. http://www.bacterio.cict.fr/p/paenibacillus.html

Felsenstein, J. (1985). Confidence limits on phylogenies: an approach using the bootstrap. Evolution 39, 783-791.

Fitch, W. M. (1971). Toward defining the course of evolution: minimum change for a specific tree topology. Syst Zool 20, 406-416.

Hespell, R. B. (1996). Fermentation of xylan, corn fiber, or sugars to acetoin and butanediol by Bacillus polymyxa strains. Curr Microbiol 32, 291-296.

Heyndrickx, M., Vandemeulebroecke, K., Scheldeman, P., Hoste, B., Kersters, K., De Vos, P., Logan, N. A., Aziz, A. M., Ali, N. \& Berkeley, R. C. W. (1995). Paenibacillus (formerly Bacillus) gordonae (Pichinoty et al. 1986) Ash et al. 1994 is a later subjective synonym of Paenibacillus (formerly Bacillus) validus (Nakamura 1984) Ash et al. 1994: emended description of P. validus. Int J Syst Bacteriol 45, 661669.

Khianngam, S., Tanasupawat, S., Lee, J.-S., Lee, K. C. \& Akaracharanya, A. (2009a). Paenibacillus siamensis sp. nov., Paenibacillus septentrionalis sp. nov. and Paenibacillus montaniterrae sp. nov., xylanase-producing bacteria from Thai soils. Int J Syst Evol Microbiol 59, 130-134.

Khianngam, S., Akaracharanya, A., Tanasupawat, S., Lee, K. C. \& Lee, J.-S. (2009b). Paenibacillus thailandensis sp. nov. and Paenibacillus nanensis sp. nov., xylanase-producing bacteria from soil. Int J Syst Evol Microbiol 59, 564-568.

Kim, D. S., Bae, C. Y., Jeon, J. J., Chun, S. J., Oh, H. W., Hong, S. G., Baek, K. S., Moon, E. Y. \& Bae, K. S. (2004). Paenibacillus elgii sp. nov., with broad antimicrobial activity. Int J Syst Evol Microbiol 54, 2031-2035.

Komagata, K. \& Suzuki, K. (1987). Lipid and cell-wall analysis in bacterial systematics. Methods Microbiol 19, 161-203.

Lee, J. C. \& Yoon, K. H. (2008). Paenibacillus woosongensis sp. nov., a xylanolytic bacterium isolated from forest soil. Int $J$ Syst Evol Microbiol 58, 612-616.

Lee, H.-J., Shin, D.-J., Cho, N. C., Kim, H.-O., Shin, S.-Y., Im, S.-Y., Lee, H. B., Chum, S. B. \& Bai, S. (2000). Cloning, expression and nucleotide sequences of two xylanase genes from Paenibacillus sp. Biotechnol Lett 22, 387-392.

Minnikin, D. E., Patel, P. V., Alshamaony, L. \& Goodfellow, M. (1977). Polar lipid composition in the classification of Nocardia and related bacteria. Int J Syst Bacteriol 27, 104-117.

Morales, P., Madarro, A., Flors, A., Sendra, J. M. \& Pérez-González, J. A. (1995). Purification and characterization of a xylanase and an arabinofuranosidase from Bacillus polymyxa. Enzyme Microb Technol 17, 424-429.

Nelson, D. M., Glawe, A. J., Labeda, D. P., Cann, I. K. O. \& Mackie, R. I. (2009). Paenibacillus tundrae sp. nov. and Paenibacillus xylanexedens sp. nov., psychrotolerant, xylan-degrading bacteria from Alaskan tundra. Int J Syst Evol Microbiol 59, 1708-1714.

Nielsen, P. \& Sørensen, J. (1997). Multi-target and mediumindependent fungal antagonism by hydrolytic enzymes in Paenibacillus polymyxa and Bacillus pumilus strains from barley rhizosphere. FEMS Microbiol Ecol 22, 183-192.

Park, M. J., Kim, H. B., An, D. S., Yang, H. C., Oh, S. T., Chung, H. J. \& Yang, D. C. (2007). Paenibacillus soli sp. nov., a xylanolytic bacterium isolated from soil. Int J Syst Evol Microbiol 57, 146-150.

Rivas, R., Mateos, P. F., Martínez-Molina, E. \& Velázquez, E. (2005a). Paenibacillus xylanilyticus sp. nov., an airborne xylanolytic bacterium. Int J Syst Evol Microbiol 55, 405-408.

Rivas, R., Mateos, P. F., Martínez-Molina, E. \& Velázquez, E. (2005b). Paenibacillus phyllosphaerae sp. nov., a xylanolytic bacterium isolated from the phyllosphere of Phoenix dactylifera. Int J Syst Evol Microbiol 55, 743-746.

Rivas, R., Garcia-Fraile, P., Mateos, P. F., Martínez-Molina, E. \& Velázquez, E. (2006). Paenibacillus cellulosilyticus sp. nov., a cellulolytic and xylanolytic bacterium isolated from the bract phyllosphere of Phoenix dactylifera. Int J Syst Evol Microbiol 56, 2777-2781.

Roux, V. \& Raoult, D. (2004). Paenibacillus massiliensis sp. nov., Paenibacillus sanguinis sp. nov. and Paenibacillus timonensis sp. nov., isolated from blood cultures. Int J Syst Evol Microbiol 54, 1049-1054.

Ruijssenaars, H. J. \& Hartsmans, S. (2001). Plate screening methods for the detection of polysaccharase producing microorganisms. Appl Microbiol Biotechnol 55, 143-149.

Saito, H. \& Miura, K. (1963). Preparation of transforming deoxyribonucleic acid by phenol treatment. Biochim Biophys Acta 72, 619-629.

Saitou, N. \& Nei, M. (1987). The neighbor-joining method: a new method for reconstructing phylogenetic trees. Mol Biol Evol 4, 406425.

Sánchez, M. M., Fritze, D., Blanco, A., Spröer, C., Tindall, B. J., Schumann, P., Kroppenstedt, R. M., Diaz, P. \& Pastor, F. I. J. (2005). Paenibacillus barcinonensis sp. nov., a xylanase-producing bacterium isolated from a rice field in the Ebro River delta. Int J Syst Evol Microbiol 55, 935-939.

Sasser, M. (1990). Identification of bacteria by gas chromatography of cellular fatty acids, MIDI Technical Note 101. Newark, DE: MIDI Inc.

Scheldeman, P., Goossens, K., Rodriguez-Diaz, M., Pil, A., Goris, J., Herman, L., De Vos, P., Logan, N. A. \& Heyndrickx, M. (2004). Paenibacillus lactis sp. nov., isolated from raw and heat-treated milk. Int J Syst Evol Microbiol 54, 885-891.

Shida, O., Takagi, H., Kadowaki, K., Nakamura, L. K. \& Komagata, K. (1997). Transfer of Bacillus alginolyticus, Bacillus chondroitinus, Bacillus curdlanolyticus, Bacillus glucanolyticus, Bacillus kobensis, and Bacillus thiaminolyticus to the genus Paenibacillus and emended description of the genus Paenibacillus. Int J Syst Bacteriol 47, 289-298.

Tamaoka, J. \& Komagata, K. (1984). Determination of DNA base composition by reversed-phase high-performance liquid chromatography. FEMS Microbiol Lett 25, 125-128. 
Tamura, K., Dudley, J., Nei, M. \& Kumar, S. (2007). MEGA 4: molecular evolutionary genetics analysis (MEGA) software version 4.0. Mol Biol Evol 24, 1596-1599.

Tanasupawat, S., Thawai, C., Yukphan, P., Moonmangmee, D., Itoh, T., Adachi, O. \& Yamada, Y. (2004). Gluconobacter thailandicus sp. nov., an acetic acid bacterium in the $\alpha$-proteobacteria. J Gen Appl Microbiol 50, 159-167.

Teather, R. M. \& Wood, P. J. (1982). Use of Congo red polysaccharide interaction in enumeration of cellulolytic bacteria from bovine rumen. Appl Environ Microbiol 43, 777-780.

Ten, L. N., Baek, S.-H., Im, W.-T., Lee, M., Oh, H. W. \& Lee, S.-T.

(2006). Paenibacillus panacisoli sp. nov., a xylanolytic bacterium isolated from soil in a ginseng field in South Korea. Int J Syst Evol Microbiol 56, 2677-2681.

Thompson, J. D., Gibson, T. J., Plewniak, F., Jeanmougin, F. \& Higgins, D. G. (1997). The CLUSTAL_X windows interface: flexible strategies for multiple sequence alignment aided by quality analysis tools. Nucleic Acids Res 25, 4876-4882.

Velázquez, E., de Miguel, T., Poza, M., Rivas, R., Rosselló-Mora, R. \& Villa, T. G. (2004). Paenibacillus favisporus sp. nov., a xylanolytic bacterium isolated from cow faeces. Int J Syst Evol Microbiol 54, 5964.

Zamost, B. L., Nielsen, H. K. \& Starnes, R. L. (1991). Thermostable enzymes for industrial applications. J Ind Microbiol 8, 71-82. 